\title{
Spherically Symmetric Bulk Viscous Fluid in Bimetric Relativity
}

\author{
S. D. Deo ${ }^{1}$, A. A. Qureshi ${ }^{2}$ \\ ${ }^{1}$ Department of Mathematics N.S.Science and Arts College Bhadrawati, Dist-Chandrapur, (M.S.) 442902, India \\ ${ }^{2}$ Department of Mathematics, Sindhu Mahavidyalaya, Nagpur-440017, India
}

Abstract: In this paper, spherically symmetric space-time is studied with bulk viscous fluid in the context of Rosen's Bimetric Theory of Relativity. Here it is shown that the bulk viscous fluid does not exist in this theory.

Keywords: Spherically symmetric, bulk viscous fluid, Bimetric Relativity

AMS Code-83C05 (General relativity)

\section{Introduction}

The bimetric theory of gravitation was proposed by Rosen[1-2](1940,1973) to modify the Einstein's general theory of relativity,by assuming two metric tensors.

In this theory he has proposed a new formulation of the general relativity by introducing a background Euclidean metric tensor $\gamma_{i j}$ in addition to the usual Riemannian metric tensor $g_{i j}$ at each point of the four dimensional space-time. With the flat background metric $\gamma_{i j}$ the physical content of the theory is the same as that of the general relativity.

Thus, now the corresponding two line elements in a coordinate system $\mathrm{x}^{\mathrm{i}}$ are -

$$
\begin{aligned}
& d s^{2}=g_{i j} d x^{i} d x^{j} \\
& \text { And } d \sigma^{2}=\gamma_{i j} d x^{i} d x^{j}
\end{aligned}
$$

Where $d s$ is the interval between two neighboring events as measured by means of a clock and a measuring rod. The interval $d \sigma$ is an abstract or geometrical quantity not directly measurable.

One can regard it as describing the geometry that would exist if no matter were present.

Spherically symmetric space-time have been studied by number of researchers Takeno[3],Gonner and Havas[ 4],Sahoo[5-6 ],Israelit[7 ],Bondi[ 8],Collins[ 9]Berger et.al[10 ]in general relativity, Whereas S.D. Deo[11,12] has studied spherically symmetric wet dark energy in Bimetric relativity. In continuation of this, we will study spherically symmetric space-time with bulk viscous fluid and will observe the result in the context of Bimetric theory of relativity.

\section{Field Equations In Bimetric Relativity}

Rosen N. has proposed the field equations of Bimetric Relativity from variation principle as

$$
\begin{aligned}
& K_{i}^{j}=N_{i}^{j}-\frac{1}{2} N g_{i}^{j}=-8 \pi \kappa T_{i}^{j} \\
& \text { Where } N_{i}^{j}=\frac{1}{2} \gamma^{\alpha \beta}\left[g^{h j} g_{h i \mid \alpha}\right]_{\mid \beta} \\
& N=N_{\alpha}^{\alpha}, \quad x=\sqrt{\frac{8}{2}} \\
& \text { and } g=\left|g_{i j}\right|, \gamma=\left|\gamma_{i j}\right|
\end{aligned}
$$

Where a vertical bar $(\mid)$ denotes a covariant differentiation with respect to $\gamma_{i j}$ 


\section{Spherically symmetric space time with bulk viscous fluid}

We consider here the spherically symmetric line element of the form

$$
d s^{2}=d t^{2}-\lambda^{2} d r^{2}-k^{2}\left(d \theta^{2}+\sin ^{2} \theta d \phi^{2}\right)
$$

Where $\lambda$ and $\mathrm{k}$ are functions of $\mathrm{r}$ and $\mathrm{t}$ only.

Corresponding to equation (3.1), we consider the line element for background metric $\gamma_{i j}$ as

$$
d \sigma^{2}=d t^{2}-\left(d r^{2}+d \theta^{2}+\sin ^{2} \theta d \phi^{2}\right)
$$

Since $\gamma_{i j}$ is the Lorentz metric i.e. $\left(-1,-1,-\sin ^{2} \theta, 1\right)$, therefore $\gamma$ - covariant derivative becomes the ordinary partial derivative.

and $T_{i}^{j}$ the energy momentum tensor for Bulk viscous fluid is given by

$$
T_{i}^{j}=(\rho+\bar{p}) v_{i} v^{j}+\bar{p} g_{i}^{j}
$$

Where $\bar{p}=p-\xi v^{j} ; i$ together with $g_{i j} v^{i} v^{j}=-1$ ie $v_{4} v^{4}=-1$ where $v_{i}$ is the four-velocity of the bulk viscous fluid $\rho, p, \bar{p}$ and $\xi$ are the energy density, isotropic pressure, effective pressure and bulk viscous coefficient.

In co-moving coordinate system we have

$$
T_{1}^{1}=T_{2}^{2}=T_{3}^{3}=\bar{p}, \quad T_{4}^{4}=-\rho \text { and } T_{i}^{j}=0 \text { for } \mathrm{i} \neq \mathrm{j}
$$

Using equations (2.1) to (2.4) with (3.1) - (3.3), we get

$$
\begin{gathered}
{\left[\left(\frac{\lambda^{\prime}}{\lambda}\right)^{\prime}-\left(\frac{\square}{\lambda}\right)^{\square}\right]-2\left[\left(\frac{k^{\prime}}{k}\right)^{\prime}-\left(\frac{\square}{k}\right)^{\square}\right]=-16 \pi \kappa \bar{p}} \\
{\left[\left(\frac{\lambda^{\prime}}{\lambda}\right)^{\prime}-\left(\frac{\square}{\lambda}\right)^{\square}\right]=16 \pi \kappa \bar{p}} \\
{\left[\left(\frac{\lambda^{\prime}}{\lambda}\right)^{\prime}-\left(\frac{\square}{\lambda}\right)^{\square}\right]+2\left[\left(\frac{k^{\prime}}{k}\right)^{\prime}-\left(\frac{\square}{k}\right)^{\square}\right]=-16 \pi \kappa \rho}
\end{gathered}
$$

And overhead primes and dots stand for ordinary differentiation with respect to coordinate $r$ and $\mathrm{t}$.

Using the field equations (3.4) - (3.6), we obtain

$$
3 \bar{p}+\rho=0
$$

In view of the reality conditions i.e. $\bar{p}>0, \rho>0$ must hold.

The above conditions (3.7) is satisfied only when

$$
\bar{p}=0, \rho=0 \text {. }
$$

Thus spherically symmetric space-time with bulk viscous fluid in bimetric relativity does not survive and hence only vacuum model is obtained.

To solve the field equations (3.4) - (3.6), we note that there are three equations connecting four unknowns, $\lambda$, $\mathrm{r}, \bar{p}$ and $\rho$. So one relation connecting these variables is needed. 
Here assume the relation between the metric potentials such as Bhattacharya and Karade

$$
k=\beta \lambda, \beta \neq 0 \text { is a constant. }
$$

Using (3.8), the vacuum field equations are

$$
\left[\left(\frac{\lambda^{\prime}}{\lambda}\right)^{\prime}-\left(\frac{\square}{\lambda}\right)^{\square}\right]=0
$$

and

$$
\left[\left(\frac{k}{k}\right)^{\prime}-\left(\frac{k}{k}\right)^{\square}\right]=0
$$

By using Method of separation of Variables, (3.10) gives us the solution

$$
\lambda=e^{\left[\frac{l}{2}\left(r^{2}+t^{2}\right)+l_{1} r+l_{2} t\right]}
$$

With the help of (3.6) we get

$$
\left.k=\beta e^{\left[\frac{l}{2}\left(r^{2}+t^{2}\right)+l_{1} r+l_{2} t\right.}\right]
$$

where, $l, l_{1}$ and $l_{2}$ are the constants of integration.

Thus substituting the value of (3.12) and (3.13) in (3.1), we get the vacuum line element as

$$
\begin{aligned}
d s^{2}=d t^{2} & -\exp 2\left[\frac{l}{2}\left(r^{2}+t^{2}\right)+l_{1} r+l_{2} t\right] d r^{2} \\
& -\beta^{2} \exp 2\left[\frac{l}{2}\left(r^{2}+t^{2}\right)+l_{1} r+l_{2} t\right]\left(d \theta^{2}+\sin ^{2} \boldsymbol{\theta} d \phi^{2}\right)
\end{aligned}
$$

\section{Conclusion}

In the study of spherically symmetric space-time, there is nil contribution of bulk viscous fluid in Bimetric theory of relativity. It is observed that bulk viscous fluid cannot be a source of gravitational field in the Rosen's bimetric theory but only vacuum model exists.

\section{Acknowledment}

The authors are thankful to Dr. K. J. Cherian,Principal,Sindhu Mahavidyalaya, Nagpur and Dr. R. D. Giri, Prof. Emeritus, P.G.T.D. (Mathematics), R. T. M. Nagpur University, Nagpur, India for their constant inspiration.

\section{References}

[1]. Rosen N. (1940) General Relativity and Flat space I. Phys. Rev.57, 147.

[2]. Rosen N. (1973) A bimetric theory of gravitation I Gen. Rela. Grav. 04, 435-47.

[3]. Takeno H.(1982) Spherically symmetric space-time in general relativity.Prog. Theo.Phys.8,317-26

[4]. Gonner H.,Havas P.(2001) Spherically symmetric space-time with constant curvature J.Maths phys 42,1063

[5]. Shahoo P.K.(2008)Spherically symmetric cosmic string in Bimetric relativity.Int.J.Theo.Phys.47,3029-3034

[6]. Shahoo P.K.(2010) Kantowski-Sachs Cosmic string in Bimetric relativityInt.J.Theo.Phys 49,25-30

[7]. Israelit M(1981)Spherical Symmetric Fields in Rosen's Bimetric Theories of Gravitation, Gen. Rela .Grav.13,681-688

[8]. Bondi H(1999) Spherical Symmetric Model in General Relativity, Gen. Rela .Grav.31,1783-1805

[9]. Collins C.B.(1985)Static Relativistic Perfect Fluid with Spherical Plane and Hyperbolic Symmetry, J.Maths.Phys. 26,2268

[10]. Berger S,Hojman R, Santamarina J.(1987)General Exact Solutions of Einstein's Equations for Static Perfect Fluid with Spherical Symmetry. J.Maths.Phys. 28,2949

[11]. Deo S.D(2013) Spherically symmetric wet dark energy in Bimetric relativity Proccedings of National conference on Frontiers of Mathematics:I Kamala Nehru Mah. Nagpur ,68-71

[12]. Deo S.D(2014) Static Spherically Symmetric Cosmological Space- Time in Bimetric Relativity IJERT Vol. 3(06), 95-97 